\title{
SPACE LATTICE FOCUSING: ON THE WAY TO EXTREMELY LOW ACCELERATED BEAM DIVERGENCE*
}

\author{
V.V.Kushin and S.V.Plotnikov" ${ }^{\#}$, ITEP, Moscow, Russia
}

\begin{abstract}
It is widely known the multiple channel acceleration is the most adequate way to save initial beam parameters due to the possibility of decreasing Coulomb forces in intensive input beams. To keep beam initial emittance and divergence for high enough specific value of the injection ion beam during acceleration the input beam should be split on multiple beams and every the micro beam must be screened from each other as much as possible. On the other hand, it is very much desirable to keep the total macro beam rather compact transversally and try to accelerate all the micro beams within the same accelerator structure at the same RF field. Attempts to use conventional quadruple focusing channels both RF and electrostatic for multiple beam acceleration usually lead to extremely complicate and bulky construction of the structure. We suppose multiple beam linac channels with alternating phase focusing (APF) as more adequate for the purpose while they are limited by less values of beam capture into acceleration process. The original version of the quadruple RF focusing multiple beam system called space lattice focusing (SLF) is supposed for getting intensive ion beam with extremely low divergence. The basic principles of the theoretical approach as well as some possible advances and restrictions for the practical use in RF linac are supposed to be discussed.
\end{abstract}

\section{INTRODUCTION}

Requirements of extremely small divergence of intensive accelerated beams may probably be successfully accomplished only by means of multiple beam accelerator systems [1]. The advantage of multiple beam approach takes maximal effect if amount of simultaneously accelerated micro beams is pretty high, i.e. some hundred or even thousands [2]. It follows from that the value of output angular divergence of total beam at given value of its total current is roughly inversely proportional to square root of number of beam channels [3]. The main purpose of multiple channel system designed for small output angular divergence is screening of every the accelerated beam from other micro beams as much as possible to avoid strong space charge forces influence on total beam emittance growth. In searching an adequate accelerating and focusing RF system we have found the principle possibility to screen multiple accelerating from each other by introducing the system of add electrodes of special form in accelerating gaps.

*Work is supported in part by ISTC

"Email: plotnikov@ vitep5.itep.ru

\section{SPACE LATTICE FOCUSING}

Let us consider the multiple beams RFQ system by using a new focusing element called "space lattice" (SLfocusing) [4]. It is well known in drift tube linac (DTL) at the edges of drift tubes particles are exposed to transversal focusing or defocusing pushes from RF field. In particular, in DTL a particle is pushed by defocusing RF field at the input and by focusing field at the output edges of drift tubes while it is roughly free from RF field action within drift tubes and accelerating gaps. We considered a possibility of substantial amplification of focusing action from RF field by increasing a number of appropriate boundary edges. It is acceptable if some additional electrodes are arranged within every accelerating gap.

By executing $M$ horizontal grooves from one flat side of a thin electrode plate to its middle and $M$ vertical grooves from the other side of this electrode again to the middle we get metal space lattice with $M \times M$ rectangular apertures with relatively thin walls in between (fig.1).

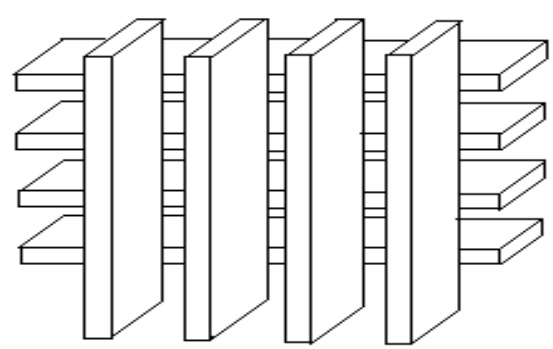

Fig.1. The fragment of Space Lattice electrode.

If several thin SLs are arranged along the common axis at the equal distances between them within accelerating gaps of the multiple channel linac we have a system which can accelerate some hundred and thousands beams simultaneously. In case of circular apertures in thin electrodes focusing and defocusing forces at both edges of thin electrodes are almost mutually compensated while in SL every particle is pushed in vertical direction at the input and in horizontal direction at the output edges even at very thin electrode depth. If we arrange $n$ SLs within an accelerating gap particles will be $n$ times pushed by focusing strength in the vertical plane and $n$ times pushed by defocusing strength in the horizontal plane. When SLs are arranged within every gap particles will be suffered some focusing action in one of transversal direction and defocusing action in other transversal direction. By turning SLs by an angle of $90^{\circ}$ in every the following gap it is possible to create a sequence of sign-alternating 
strengths analogous to field strengths at spatially uniform RFQ focusing.

\section{TRANSVERSAL STABILITY IN SLF ARRAYS}

Let us consider basic aspects of transversal stability of ion motion in SLF arrays. The analysis of the system properties is carried out for a single cell (channel) of SL electrodes according to the approach presented in [5].

$\mathrm{RF}$ field potential within the acceleration gap has the planes of symmetry $\mathrm{XOZ}$ and $\mathrm{YOZ}$ :

$$
U(x, y, z)=U(x,-y,-z)=U(x,-y, z) \text {. }
$$

By introducing the function of $G(x, y, z)$ which defines the level of deviation from axial symmetry in linear approximation and is responsible for quadruple focusing effect

$$
G(x, y, z)=\frac{1}{2}\left(\frac{\partial E_{x}}{\partial x}-\frac{\partial E_{y}}{\partial y}\right) .
$$

and taking into account the condition of mutual connection of electric field components $\operatorname{div} E=0$, i.e.

$$
\frac{\partial E_{x, y}}{\partial x, y}=-\frac{1}{2} \frac{\partial E_{z}}{\partial z} \pm G,
$$

in linear approximation the transversal motion equations may be written in the form:

$$
\begin{aligned}
& \frac{d^{2} x}{d t^{2}}=\frac{z e}{m_{0}} \frac{\partial E_{x}(z)}{\partial x} x \cdot \cos \omega t(z), \\
& \frac{d^{2} y}{d t^{2}}=\frac{z e}{m_{0}} \frac{\partial E_{y}(z)}{\partial y} y \cdot \cos \omega t(z),
\end{aligned}
$$

Assuming the conditions of $x=$ const, $y=$ const are valid within SL electrodes we can write the expressions for transversal velocity increments in thin lenses approximation:

$$
\begin{aligned}
& \Delta \frac{d x}{d t}=\frac{z e x}{m_{0}} \int_{-d / 2}^{d / 2} \frac{\partial E_{x}(z)}{\partial x} \cdot \frac{d z}{v} \cos \omega t(z), \\
& \Delta \frac{d y}{d t}=\frac{z e y}{m_{0}} \int_{-d / 2}^{d / 2} \frac{\partial E_{y}(z)}{\partial y} \cdot \frac{d z}{v} \cos \omega t(z),
\end{aligned}
$$

Assuming ion velocity is constant within RF quadruples and going to the independent variable of $\tau=v t / S$ ( $S$ is the length of focusing period), the expressions for transversal velocity increments may be presented in the form of

$$
\begin{gathered}
\Delta \frac{d x}{d \tau}=\left(b_{1}+b_{2}\right) x, \quad \Delta \frac{d y}{d \tau}=\left(b_{1}-b_{2}\right) y, \\
b_{1}=\frac{z e S}{2 m_{0} v^{2}} \int_{-d / 2}^{d / 2} \frac{\partial E_{z}}{\partial z} \cos \omega t(z) d z \\
b_{2}=\frac{z e S}{2 m_{0} v^{2}} \int_{-d / 2}^{d / 2} G(z) \cos \omega t(z) d z
\end{gathered}
$$

Let us consider now the system of SL electrodes arranged according to that presented in Fig.2.



Fig.2. Structure of multiple beam array with $S=\beta \lambda$.

By splitting the focusing period into segments we can get the matrix of the period by means of multiplication matrixes of all the segments.
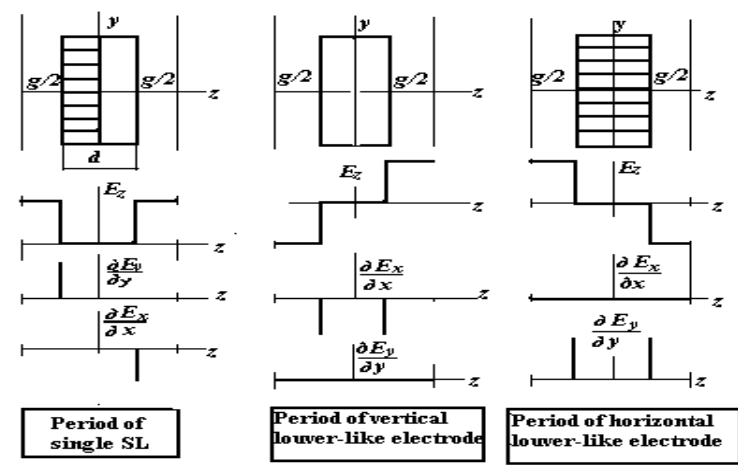

Fig.3. Possible segments of focusing elements (a - space lattice, b - vertical louver, c- horizontal louver).

The segment with a single cell of SL electrode is presented in fig. $3 \mathrm{a}$.

The coefficient $G(z)$ changes its sign within this segment.

$$
G(z)=\frac{1}{2} \frac{\partial E_{z}}{\partial z}, z<0, \quad G(z)=-\frac{1}{2} \frac{\partial E_{z}}{\partial z}, z>0 .
$$

As a result of the integrating along the SL electrode length the coefficients $b_{1}$ and $b_{2}$ may be written as

$$
\begin{gathered}
b_{1}=p_{0} \sin \frac{\pi d}{\beta \lambda} \sin \varphi, \quad b_{2}=-p_{0} \cos \frac{\pi d}{\beta \lambda} \cos \varphi, \\
p_{0}=\frac{z e S}{2 W} E_{0} .
\end{gathered}
$$

In these expressions $p_{0}$ is pick value of RF field focusing. The increments of transversal velocity may be written in the form ( $d$ is the length of SL electrode):

$$
\Delta \frac{d x}{d \tau}=-p_{0} \cos \left(\varphi+\frac{\pi d}{\beta \lambda}\right) x, \quad \Delta \frac{d y}{d \tau}=p_{0} \cos \left(\varphi-\frac{\pi d}{\beta \lambda}\right) y .
$$

Coming to the thin lens approximation $(d / \beta \lambda<<1)$ the matrixes of focusing element may be written as: 


$$
\begin{gathered}
p=\left[\begin{array}{ll}
1 & 0 \\
p & 1
\end{array}\right] \quad \bar{p}=\left[\begin{array}{cc}
1 & 0 \\
\bar{p} & 1
\end{array}\right] \\
p=-\bar{p}=p_{0} \cos \varphi .
\end{gathered}
$$

The matrixes of the segment presented in fig. $3 \mathrm{a}$ correspond to motions in $\mathrm{XOZ}$ and $\mathrm{YOZ}$ planes.

$$
\begin{aligned}
& T_{n x}=\left[\begin{array}{cc}
1 & \frac{1}{4\left(N_{d}+1\right)} \\
0 & 1
\end{array}\right]\left[\begin{array}{cc}
1 & 0 \\
p_{0} \cos \varphi_{n} & 1
\end{array}\right]\left[\begin{array}{cc}
1 & \frac{1}{4\left(N_{d}+1\right)} \\
0 & 1
\end{array}\right] \\
& T_{n y}=\left[\begin{array}{cc}
1 & \frac{1}{4\left(N_{d}+1\right)} \\
0 & -1
\end{array}\right]\left[\begin{array}{cc}
1 & 0 \\
-p_{0} \cos \varphi_{n} & 1
\end{array}\right]\left[\begin{array}{cc}
1 & \frac{1}{4\left(N_{d}+1\right)} \\
0 & 1
\end{array}\right]
\end{aligned}
$$

Here $\varphi_{n}$ is a phase of particle in the $n^{\text {th }} \mathrm{SL}$ of focusing period.

For the segment with vertical orientation of through slots or vertical "louver"(VL) (see fig.3b) the coefficient $G(z)$ does not change its sign):

$$
G(z)=-\frac{1}{2} \cdot \frac{\partial E_{z}}{\partial z}
$$

Such electrodes are usually arranged at one of edges of acceleration period. Electrodes with horizontal orientation of through slots (HL) are arranged at the other edge of acceleration period (see fig. $3 \mathrm{c}$ ).

The coefficients of $b_{1}$ and $b_{2}$ are equal to each other:

$$
b_{1}=b_{2}=b=-p_{0} \cos \varphi \cos (\pi d / \beta \lambda) \text {. }
$$

Moving through the electrode with vertical orientation of slots a particle gains double focusing momentum from $\mathrm{RF}$ field in $X$ direction while is not pushed in $Y$ direction

$$
\Delta \frac{d x}{d \tau}=-2 p_{0} \cos \varphi, \quad \Delta \frac{d y}{d \tau}=0 .
$$

For the edge electrodes arranged $\beta \lambda 2$ apart from these electrodes the through slots are oriented horizontally so particles gain double momentum from RF field in $Y$ direction while no pushed in $X$ direction.

The analytical calculations have been carried out assuming that focusing effect is proportional to $p_{0}^{2}$; the defocusing effect is proportional to $p_{0}$ while higher powers of $p_{0}$ were not taken into account.

In fig.4 the results of matrix analysis are plotted for the case of $p_{0}=1$. It is seen that in assumption of zero length of SLs at arrangement of 12 focusing elements within the focusing period all particles are stable in transversal directions independently on their input phase. It is also follows from comparison analytical and matrix calculation results that focusing action of focusing lattices is proportional to the coefficient at $p_{0}^{2}$ with rather high accuracy.

The focusing effect is weakened to some extent with allowance for finite width of SLs. It was found for the case when lattice electrodes occupy one half of focusing period the necessary number of focusing SLs at the period is about $30 \%$ more than in case of zero-width SLs to make transversal stability available for any input phase of a particle.



Fig.4. Phase advance vs. input phase of ion ( $\mathrm{N}$ is amount of space lattices within an acceleration period of $\beta \lambda / 2$ ).

The presence of both grooves and slots distorts to some extent the field structure near the electrode borders. To increase focusing features of the system it is necessary to decrease field penetration into focusing elements by narrowing both grooves and slots. The compromise is probably corresponds to the case of field strength falling to zero in the middle of focusing electrodes.

The preliminary analysis shows that by arranging of appropriate amount of SLs in every the acceleration gap the considering multiple channel focusing acquires features of the spatially uniform RF quadruple focusing. Theoretically any small beam divergence of total accelerated beam may be reached in SLF array while features of practical use of SL focusing in RF linacs seem to be established by adequate ion injectors as well as advanced level of technology and practice.

\section{REFERENCES}

[1] B.P.Murin, B.I.Bondarev, V.V.Kushin, A.P.Fedotov. Ion Linear Accelerators, Moscow, Atomizdat, p.207 (1978).

[2] A.W.Maschke "MEQALAC: A New Approach to Low Beta RF Acceleration", BNL 51029 (1979).

[3] V.V.Kushin et al, "First Experience of Works With Compact Injectors For Trials and Drills of RF Linac Structures", XIX Intern. Linac Conf. LINAC98, Chicago, (1998), to be published

[4] E.V.Gromov, V.V.Kushin, S.V.Plotnikov, «Multiple Channel Structure For Ion Linac», Inventor's Certificate of USSR № 256492 (1986); and S.V.Plotnikov «Operating variables of RF focusing in linac structures», Thesis, 2 (1986) (in Russian)

[5] I.M.Kapchinsky, "Theory of Linear Resonant Accelerators", Atomizdat, Moscow, (1966), Harwood, 1985. 